\title{
Tecnologia assistiva no contexto dos cursos de biblioteconomia do Norte e Nordeste do brasil
}

\section{Assistive technology in the context of librarianship courses in the North and Northeast of brazil}

Cenidalva Miranda de Souza Teixeira ${ }^{1 *}$, Silvana Maria de Jesus Vetter ${ }^{1}$, Vanessa Thalyane Pereira Ferreira ${ }^{1}$, Jennyfer de Oliveira Diniz ${ }^{1}$, Lucas da Silva Araújo ${ }^{1}$

\section{RESUMO}

Estudo descritivo sobre o uso de Tecnologia Assistiva nas Instituições de Ensino Superior públicas das regiões Norte e Nordeste do Brasil. Objetiva analisar a presença desta temática nos cursos de Biblioteconomia das IES públicas do Norte e Nordeste. Adota como metodologia as pesquisas: bibliográfica, documental, e de campo, com abordagem quanti-qualitativa na análise dos dados. Discorre sobre conceitos relacionados à tecnologia assistiva, enfatizando sua importância na promoção dos direitos das pessoas com deficiência. Contribui para a disseminação do conhecimento sobre tecnologia assistiva, bem como o seu uso nos cursos de Biblioteconomia das instituições de ensino superior públicas do Norte e Nordeste brasileiro.

Palavras-chave: Acessibilidade; Tecnologia Assistiva; Educação Inclusiva; Biblioteconomia; Instituições de Ensino Superior Públicas - Norte e Nordeste - Brasil

\begin{abstract}
Descriptive study on the use of Assistive Technology in public Higher Education Institutions in the North and Northeast regions of Brazil. It aims to analyze the presence of the theme in the Librarianship courses of public HEIs in the North and Northeast. It adopts research as a methodology: bibliographic, documentary, and field with quantitative-qualitative approach in data analysis. It discusses concepts related to assistive technology emphasizing its importance in promoting the rights of people with disabilities. It contributes to the dissemination of knowledge about assistive technology, as well as its use in Library Science courses at public higher education institutions in the North and Northeast of Brazil.
\end{abstract}

Keywords: Accessibility. Assistive Technology. Inclusive Education. Librarianship. Public Higher Education Institutions - North and Northeast - Brazil

\footnotetext{
${ }^{1}$ Universidade Federal do Maranhão. *E-mail: cenidalva@gmail.com
} 


\title{
INTRODUÇÃO
}

Acessibilidade é uma temática de suma importância, uma vez que se trata de um dos direitos fundamentais das pessoas com deficiência. No Brasil, segundo Wagner et. al. (2010), discussões envolvendo o assunto tiveram início por volta de 1990 no meio acadêmico.

\begin{abstract}
Apesar de recente, esta é uma área de estudo em crescimento no país, porém, existe um longo caminho a ser percorrido para que se consolide, de modo a desconstruir os estereótipos arraigados no imaginário popular, como, por exemplo, a restrição do termo acessibilidade à ideia de acesso físico de cadeirantes possibilitado por rampas [...] (FERREIRA, 2019, p. 19).
\end{abstract}

Além das barreiras físicas, a acessibilidade também envolve barreiras urbanísticas, arquitetônicas, nos transportes, na comunicação e informação, atitudinais e tecnológicas. A existência destas barreiras pode não somente dificultar a vida das pessoas com deficiência, como também impossibilitar a realização de suas tarefas básicas, criando a falsa ideia de deficiência como sinônimo de incapacidade.

As discussões sobre acessibilidade são importantes para a desconstrução desta ideia, pois a dificuldade, ou até mesmo, a impossibilidade da execução de algumas tarefas por pessoas com deficiência são consequência dos obstáculos nos ambientes, não estando relacionado à incapacidade. Estudos na área também são relevantes para a criação de tecnologias acessíveis, e em âmbito social, para a conscientização das pessoas.

O aumento da quantidade de pesquisas sobre a temática é importante, pois a efetivação dos direitos das pessoas com deficiência deve ser tratada com prioridade, assim como a realização e publicação de tais pesquisas. Estes estudos podem contribuir para combater o desrespeito e descaso em relação aos direitos destes cidadãos, pois a partir de percepções diárias é possível identificar as falhas na prática da acessibilidade e, deste modo, aplicar soluções.

A pesquisa neste campo contribui para reflexões pela comunidade acadêmica em relação à necessidade de estudos sobre o tema, abrindo ainda um leque de possibilidades para futuros estudos. Para Biblioteconomia, a pesquisa reflete no fortalecimento de seus aspectos humanístico e social, possibilitando também aos docentes refletirem sobre a necessidade de sua capacitação na área para uma melhor atuação em sala de aula.

Nesse sentido, delineou-se como objetivo geral: analisar, nos cursos de Biblioteconomia das Instituições de Ensino Superior públicas das regiões Norte e 
Nordeste do Brasil, a presença do tema Tecnologia Assistiva, considerando-se a indispensabilidade destas ferramentas ao atendimento satisfatório das necessidades informacionais da pessoa com deficiência.

Elencou-se como objetivos específicos: identificar os principais conceitos relacionados ao campo da Tecnologia Assistiva; apresentar a importância da Tecnologia Assistiva no contexto acadêmico; e refletir sobre a abordagem do tema Tecnologia Assistiva nas disciplinas dos cursos de Biblioteconomia das IES públicas do Norte e Nordeste brasileiro.

Este trabalho está organizado em cinco seções, iniciadas pela introdução. $\mathrm{Na}$ segunda seção, detalha-se a metodologia adotada no decorrer da pesquisa. Na terceira seção, apresenta-se o conceito de Tecnologia Assistiva e sua relevância no Ensino Superior. Na quarta seção, descreve-se os resultados da pesquisa de campo analisando a presença da Tecnologia Assistiva nos cursos de Biblioteconomia das IES públicas do Norte e Nordeste. Na última seção, faz-se as considerações finais sobre a pesquisa.

\section{METODOLOGIA}

A pesquisa se caracteriza como exploratória, pois buscou-se familiarização com o problema de modo a esclarecê-lo (GIL, 2002), e descritiva, uma vez que não houve interferência do pesquisador, apenas a descrição das características do objeto de pesquisa (BARROS; LEHFELD, 2007).

O primeiro passo para a realização da pesquisa foi a identificação de fontes de informação que tratam do tema para embasamento teórico. A busca foi feita na internet por meio do buscador Google, sendo usadas as como palavras-chave: acessibilidade, tecnologias acessíveis, Tecnologia Assistiva, educação especial, educação inclusiva etc.

Após a identificação das fontes, fez-se pré-leitura das obras para selecionar os documentos que poderiam compor a fundamentação do estudo. Foram examinados a folha de rosto, o sumário, os índices, as referências bibliográficas, as notas ao pé da página, o prefácio, a introdução e a conclusão (CERVO; BERVIAN; SILVA, 2007).

O passo seguinte foi a leitura seletiva com o olhar voltado ao problema formulado e as perguntas elaboradas quando se questionou o assunto, ou seja, os objetivos do trabalho. Foram identificadas e selecionadas informações que pudessem fornecer esclarecimentos sobre o problema (CERVO; BERVIAN; SILVA, 2007). 
A seleção está relacionada à pesquisa bibliográfica, que é um dos primeiros passos em uma investigação. De acordo com Marconi e Lakatos (2003, p. 183), a pesquisa bibliográfica tem por objetivo "[...] colocar o pesquisador em contato direto com tudo o que foi escrito, dito ou filmado sobre determinado assunto [...]".

Deste modo, fez-se a identificação dos principais autores que investigam o tema Tecnologia Assistiva, as principais discussões relacionadas a esse campo, bem como o seu papel na inclusão de pessoas com deficiência no Ensino Superior. Esta pesquisa também serviu para nortear a análise dos resultados encontrados.

Este estudo inclui ainda pesquisa documental, ou seja, aquela realizada a partir de fontes primárias. Foi feito o uso de documentos como o Projeto Político Pedagógico dos cursos de Biblioteconomia da IES públicas do Norte e Nordeste, a fim de obter informações que contribuíssem para a construção do instrumento de coleta de dados.

Depois da seleção dos documentos nas pesquisas bibliográfica e documental, foram feitas as leituras reflexiva e interpretativa. Na leitura reflexiva foram identificadas ideias principais e secundárias, determinando-se, com base nos objetivos da pesquisa sua utilidade (CERVO; BERVIAN; SILVA, 2007). Na leitura interpretativa, as ideias identificadas anteriormente foram aplicadas aos fins específicos do estudo, embasando as ideias do pesquisador.

O próximo passo foi a coleta de dados, que, segundo Barros e Lehfeld (2007), consiste na fase na qual a realidade é indagada, e que por meio da aplicação de técnicas, dados são obtidos. Nessa fase foram determinados a população de estudo e o instrumento de coleta de dados, assim como a programação da coleta (CERVO; BERVIAN; SILVA, 2007).

A pesquisa de campo foi realizada nas IES públicas do Norte e Nordeste do Brasil, mas por questões geográficas, os dados foram coletados por meio da ferramenta Google Forms, que possibilita a criação de questionários com perguntas abertas, fechadas ou mistas. Para este estudo foi criado um questionário misto, sendo o link enviado por email.

O questionário foi enviado aos professores dos cursos de Biblioteconomia das 13 (treze) Instituições Públicas de Ensino Superior do Norte e Nordeste, havendo respostas representando 11 (onze) destas instituições, a saber: as Universidades Federais do Maranhão, do Rio Grande do Norte, de Alagoas, da Paraíba, do Cariri, de Rondônia, de Sergipe, do Ceará, do Amazonas, da Bahia, e a Universidade Estadual do Piauí. 
O último passo foi a análise dos dados, na qual se adotou a abordagem quantiqualitativa, uma vez que além da quantificação dos resultados, foi feita a compreensão do objeto estudado, produzindo-se informações detalhadas sobre o mesmo.

\section{TECNOLOGIA ASSISTIVA NO ENSINO SUPERIOR}

Tecnologia Assistiva é um campo interdisciplinar que envolve o estudo e desenvolvimento de ferramentas que contribuem para a promoção da acessibilidade às pessoas com deficiência, considerando as limitações impostas pelos ambientes nos quais estão inseridas, bem como suas necessidades individuais. Além disso, essas ferramentas são elementares no que diz respeito ao processo de inclusão destes cidadãos na sociedade.

Esses produtos são importantes para o fortalecimento do processo de inclusão social, englobando fatores determinantes para garantir qualidade de vida às pessoas com deficiência. $\mathrm{O}$ uso desses recursos tecnológicos é necessário para a eliminação dos obstáculos que reduzem o desempenho funcional na execução de atividades básicas. Essas ferramentas podem ajudar na superação das barreiras urbanísticas, arquitetônicas, nos transportes, na comunicação e informação, e sobretudo, tecnológicas [...] (FERREIRA, 2019, p. 30).

Como se pode perceber, os produtos de Tecnologia Assistiva são essenciais não somente para o processo de inclusão social, como também para proporcionar uma vida mais digna a esses cidadãos ao lhes possibilitar a execução de tarefas básicas do cotidiano. Fora que esses produtos não estão restritos ao acesso físico, pois envolvem todas as dimensões da acessibilidade, promovendo, por exemplo, os direitos de ir e vir e de acesso a informação.

Os recursos de Tecnologia Assistiva são uma alternativa interessante de ser adotada em prol da inclusão de pessoas com deficiência no Ensino Superior, possibilitando uma efetiva educação para todos. No entanto, promover uma educação inclusiva no ensino terciário depende não apenas de se garantir o acesso, mas também de se assegurar a permanência dos estudantes com deficiência até concluírem sua graduação.

Além da permanência, o aprendizado e o desempenho acadêmico destes estudantes é resultado das condições que lhe são ofertadas pelas Instituições de Ensino Superior (POLIA et al., 2013). Sendo assim, a promoção da acessibilidade é um passo essencial, mas por si só não é suficiente, pois conforme afirmam Furtado e Leal (2014), o aperfeiçoamento do currículo e a qualificação dos professores também são investimentos necessários. 
O uso de Tecnologia Assistiva é tão fundamental quanto a acessibilidade arquitetônica e comunicacional, a formação continuada dos docentes, e a mediação pedagógica (POLIA et al., 2013). Embora exista a questão da pouca quantidade de ferramentas de Tecnologia Assistiva ofertadas no mercado e de seu alto custo, conta-se ainda com soluções simples, a partir do uso de conhecimento e recursos disponíveis gratuitamente ou a baixo custo.

A adoção dessas ferramentas, portanto, não depende exclusivamente de recursos financeiros, nem tampouco implica na necessidade de altos investimentos. Muitas vezes, é preciso criatividade e disposição para a identificação de alternativas para cada caso (FURTADO; LEAL, 2014). Embora esses dois elementos sejam importantes, é necessário também o desenvolvimento de habilidades para o uso de Tecnologia Assistiva.

De acordo com Seeger, Henn e Morgenstern (2020), essas ferramentas só podem ser bem aproveitadas se os envolvidos as usarem como meio de interação, buscando aquisição de habilidades. Em complemento, Toso et al. (2018) afirmam que para que esses recursos tecnológicos sirvam como auxílio no processo de inclusão, as pessoas precisam se interessar pela busca do aperfeiçoamento, a fim de que aprendam a manuseálos.

A aquisição de habilidades referentes ao uso destes recursos acessíveis e gratuitos é possível por meio da prática, tanto de estudantes como de professores, orientada por manuais e tutoriais disponíveis na internet. Outro ponto de destaque no que diz respeito à presença da Tecnologia Assistiva nas salas de aula das Instituições de Ensino Superior, é o incentivo ao desenvolvimento de pesquisas sobre a temática.

Como apontam Varela e Oliver (2012), essas pesquisas são relevantes ao justificarem a importância da Tecnologia Assistiva, e caracterizarem a utilização destas no dia a dia das pessoas com deficiência, reforçando os argumentos relacionados ao cumprimento dos direitos destes cidadãos. As pesquisas contribuem ainda para o combate de práticas discriminatório, por meio da conscientização social.

O setor acadêmico tem papel fundamental na pesquisa, no desenvolvimento e na disponibilização de produtos de Tecnologia Assistiva, como destacam Rodrigues e Alves (2013). Posto isto, é possível se perceber a pertinência do tema no processo de formação de profissionais dos diversos ramos do conhecimento, não ficando restringido aos campos de Terapia Ocupacional e Educação Inclusiva. 


\section{TECNOLOGIA ASSISTIVA NOS CURSOS DE BIBLIOTECONOMIA DAS IES DO NORTE E NORDESTE}

A análise dos dados coletados durante a fase da pesquisa de campo proporcionou a obtenção de um panorama relacionado à utilização e conhecimento no que diz respeito à Tecnologia Assistiva nos Cursos de Biblioteconomia da Instituições de Ensino Superior Públicas das Regiões Norte e Nordeste do Brasil, conforme apresentado a seguir.

Foram analisados questionários representando 11 (onze) das IES públicas do Norte e Nordeste brasileiro. O questionário foi configurado para direcionar os participantes a questões distintas, levando em consideração se as disciplinas contemplam o tema de TA, e se existe aplicação prática deste conteúdo em sala de aula.

Menos de $30 \%$ dos participantes da pesquisa ministram disciplinas que contemplam a temática de Tecnologia Assistiva, o que pode estar relacionado com o fato de que, na maioria das vezes, as discussões sobre este tema ficam restritas ao grupo de profissionais que atuam especificamente em áreas relacionadas às pessoas com deficiência, como problematizado por Rodrigues e Alves (2013). Esses dados chamam atenção, uma vez que é elementar que o tema esteja presente nas discussões de todos os campos do conhecimento.

Os professores que ministram disciplinas que contemplam a TA foram direcionados a mais seis questões. A primeira questão foi sobre a quantidade de horas disponibilizadas na disciplina para tratar sobre Tecnologia Assistiva. Apesar da variação nas respostas, percebeu-se que há uma conscientização, identificada por Rodrigues e Alves (2013), sobre a importância de se ter um tempo dedicado especialmente para as discussões sobre o tema em sala de aula

Outra questão foi relacionada a forma como a disciplina é trabalhada durante as aulas, sendo que em $75 \%$, esta é somente teórica. A maioria das justificativas quanto a não realização da prática está relacionada a falta de recursos, o que expressa desconhecimento sobre opções sem custo que podem ser adotadas como uma alternativa, usando-se a criatividade para superar a falta de recursos financeiros e materiais, como explanado por Furtado e Leal (2014).

Os $25 \%$ que realizam a prática na disciplina utilizam recursos virtuais, digitais e eletrônicos disponíveis gratuitamente na internet, e em um dos casos, equipamentos e espaços (como laboratório) da universidade. Esses dados evidenciam a possibilidade de 
se oferecer condições para a prática que não necessariamente dependem de investimentos financeiros ou recursos exclusivamente disponibilizados pela Instituição de Ensino Superior.

Em relação à importância de o tema ser trabalhado em sala de aula, as respostas incluem tópicos como inclusão, democratização do conhecimento, preparação do estudante ao mercado de trabalho, estímulo ao desenvolvimento tecnológico e social, e conscientização quanto a diversidade. Tudo isso é importante para que o estudante esteja apto para buscar as informações necessárias ao exercício de seu senso crítico e formação de opinião, bem como fazer intervenções no seu meio, como discorrido por Toso et al. (2018).

No que diz respeito as dificuldades encontradas ao abordar o tema, a maioria das respostas envolve questões práticas, destacando-se a falta de recursos de Tecnologia Assistiva disponibilizados pelas instituições, o que pode ser revertido por meio do uso de recursos gratuitos. Destacou-se ainda a falta de capacitação dos profissionais, mas como salientado por Toso et al. (2018), é importante que as pessoas tenham interesse para buscar o aperfeiçoamento para o manuseio destes recursos, adquirindo, assim, as habilidades necessárias.

Mais de $60 \%$ dos participantes que ministram disciplinas que contemplam a temática acreditam que seja necessário ter uma disciplina específica sobre Tecnologia Assistiva, o que mostra a consciência destes profissionais sobre a importância do tema, contando-se com mais tempo para as discussões. A partir de uma disciplina específica é possível aproveitar melhor os elementos que surgem para a produção de novos conhecimentos (TOSO et al., 2018), contribuindo deste modo para o desenvolvimento desta área.

Aos mais de $70 \%$ dos participantes que ministram disciplinas que não contemplam o tema de TA foram feitas outras duas perguntas. A primeira questão foi referente ao porquê desta temática não está inserida no conteúdo da disciplina, a maioria das respostas está relacionada ao fato de que estas disciplinas não contemplam o tema, indicando a indispensabilidade de aperfeiçoamento dos currículos, como exposto por Furtado e Leal (2014).

Algumas das justificativas para a ausência do tema nas disciplinas estão relacionadas à falta de iniciativa do professor, destacando-se uma que manifesta a necessidade de um debate com Núcleo Docente Estruturante, Colegiado e Coordenação 
de curso, exprimindo não caber apenas ações individuais dos docentes. Considerando-se, portanto, que um dos papéis fundamentais no processo de inclusão é a mediação pedagógica, como apontado por Polia et al. (2013), tendo ou não o professor autonomia para ações individuais, este pode atuar na mediação entre os atores que farão parte de todo o processo inclusivo.

Ao serem indagados sobre a melhor forma de se introduzir o tema em sala de aula, a maioria dos participantes optou pelas disciplinas de tecnologia, o que ressalta o impacto que o avanço tecnológico tem no processo de inclusão das pessoas com deficiência. Por outro lado, esses dados também revelam uma percepção limitada sobre a relevância de discussões que envolvem Tecnologia Assistiva em todos os âmbitos, de modo a assegurar a concretização deste conceito, como defendido por Rodrigues e Alves (2013).

Outra opção que se destacou foi a introdução do tema em todas as disciplinas, o que corrobora com a imprescindibilidade de que todas as áreas discutam sobre o assunto, buscando uma integração de saberes para a promoção de ações que combatam a exclusão social, conforme a visão de Rodrigues e Alves (2013). Destarte, ressalta-se que o tema não só pode como deve ser abordado nas demais disciplinas, além das tradicionalmente relacionadas à área.

\section{CONCLUSÃO}

A pesquisa teve como objetivo geral analisar a presença do tema Tecnologia Assistiva (TA) nos cursos de Biblioteconomia das IES públicas das regiões Norte e Nordeste do Brasil. Refletindo-se sobre esta finalidade, elencou-se os seguintes objetivos específicos: identificar os principais conceitos relacionados ao campo da TA; apresentar a importância da TA no contexto acadêmico; e refletir sobre a abordagem do tema TA nas disciplinas dos cursos de Biblioteconomia das IES públicas do Norte e Nordeste brasileiro.

Os principais conceitos referentes ao campo da Tecnologia Assistiva identificados durante a pesquisa bibliográfica foram: acessibilidade, que envolve ações para a superação de barreiras físicas, informacionais e tecnológicas; e inclusão, movimento que busca promover a participação efetiva de todas as pessoas na sociedade, combatendo preconceitos e atitudes discriminatórias. Notou-se a importância da tecnologia tanto na promoção da acessibilidade quanto na consolidação do processo de inclusão das pessoas com deficiência. 
No que diz respeito à importância da Tecnologia Assistiva nas Instituições de Ensino Superior, verificou-se três pontos pertinentes: o uso dos recursos de TA para garantir a permanência e melhorar o aprendizado do estudante com deficiência em sua graduação; a presença do tema em discussões acadêmicas e o incentivo a pesquisas na área que contribuem, sobretudo, para o desenvolvimento de mais recursos; e a abordagem do tema em sala aula proporcionando uma formação mais completa de futuros profissionais.

Em relação à abordagem de Tecnologia Assistiva nas disciplinas dos cursos de Biblioteconomia das IES públicas do Norte e Nordeste brasileiro, observou-se que o tema ainda é contemplado em poucas disciplinas. Apesar disso, no geral, é notório a existência de uma consciência sobre a importância da área para o processo de inclusão das pessoas com deficiência não somente no Ensino Superior, mas em todos os âmbitos.

Destaca-se também na pesquisa a falta de recursos de Tecnologia Assistiva como justificativa para a ausência do tema ou da prática em sala de aula e, até mesmo, como uma das dificuldades enfrentadas ao se trabalhar com o assunto. A busca pelo conhecimento e recursos disponíveis gratuitamente surge, assim, como uma alternativa viável para a superação deste obstáculo, o que possibilitará a construção de uma comunidade acadêmica acolhedora.

Espera-se que este trabalho contribua para o campo da Tecnologia Assistiva através da conscientização sobre a importância deste tema, principalmente, no que se refere a construção de uma sociedade inclusiva, e também incentive a realização de mais pesquisas na área.

\section{REFERÊNCIAS}

BARROS, Aidil Jesus da Silveira; LEHFELD, Neide Aparecida de Souza. A pesquisa e a iniciação científicas. In: BARROS, Aidil Jesus da Silveira; LEHFELD, Neide Aparecida de Souza. Fundamentos de Metodologia Científica. 3. ed. São Paulo: Pearson Prentice Hall, 2007. cap. 7, p. 105-118.

CERVO, Amado Luiz; BERVIAN, Pedro Alcino; SILVA, Roberto da. Elaboração e comunicação da pesquisa. In: CERVO, Amado Luiz; BERVIAN, Pedro Alcino; SILVA, Roberto da. Metodologia Científica. 6. ed. São Paulo: Pearson Prentice Hall, 2007. cap. 6, p. 71-90.

FERREIRA, Vanessa Thalyane Pereira. O uso de softwares de Tecnologia Assistiva nas bibliotecas universitárias federais da região Nordeste do Brasil. Orientadora: Cenidalva Miranda de Sousa Teixeira. 2019. 61f. Trabalho de Conclusão de Curso (Bacharelado em Biblioteconomia) - Universidade Federal do Maranhão, São Luís, 
2019. Disponível em:

https://monografias.ufma.br/jspui/bitstream/123456789/4068/1/Vanessa\%20Thalyane\% 20P.F..pdf. Acesso em: 17 set. 2020.

FURTADO, Seyla Cristhina Silvestre; LEAL, Zaira Fátima de Rezende Gonzalez. A tecnologia Assistiva na formação do professor: um estudo em escola especial. In: OS DESAFIOS da escola pública paranaense na perspectiva do professor PDE: artigos, Cadernos PDE, v.1, 2014. Disponível em:

http://www.diaadiaeducacao.pr.gov.br/portals/cadernospde/pdebusca/producoes_pde/20 14/2014_uem_edespecial_artigo_seyla_cristhina_silvestre.pdf. Acesso em: 25 set. 2020.

GIL, Antônio Carlos. Como classificar as pesquisas? In: GIL, Antônio Carlos. Como elaborar projeto de pesquisa. 4 ed. São Paulo: Atlas, 2002. cap.4, p. 41-58.

MARCONI, Marina de Andrade. LAKATOS, Eva Maria. Técnicas de pesquisa. In: MARCONI, Marina de Andrade. LAKATOS, Eva Maria. Fundamentos de Metodologia Científica. 5 ed. São Paulo, Atlas: 2003. cap. 9, p. 174-214.

POLIA, Andreza Aparecida et al. O papel da Tecnologia Assistiva no Ensino Superior: um olhar às possibilidades e dificuldades. In: SIMPÓSIO INTERNACIONAL DE ESTTUDOS SOBRE DEFICIÊNCIA, 1., 2013, São Paulo. Anais eletrônicos [...]. São Paulo: USP, 2013. Disponível em:

http://www.memorialdainclusao.org.br/ebook/Textos/Maria_da_Conceicao_Narkhira_P ereira_Pedrosa.pdf. Acesso em: 26 set. 2020.

RODRIGUES, Patrícia Rocha; ALVES, Lynn Rosalina Gama. Tecnologia Assistiva: uma revisão do tema. Holos, ano 29, v. 26, 2013, p. 170-180. Disponível em: http://200.9.65.226/bitstream/fieb/687/1/Tecnologia\%20assistiva\%20\%20uma\%20revis\%c3\%a3o\%20....pdf. Acesso em: 21 set. 2020.

SEEGER, Mariza Gorette; HENN, Leonardo Guedes; MORGENSTERN, Juliane Marschall. Tecnologias Assistivas: reflexões sobre as práticas formativas inclusivas no Ensino Superior. Textura, v. 22, n. 51, 2020, p. 292-316. Disponível em: http://www.periodicos.ulbra.br/index.php/txra/article/view/5401. Acesso em: 26 set. 2020.

TOSO, Carine et al. A tecnologia assistiva no ensino superior: reflexões sobre seu uso para alunos ouvintes e surdos. Revista On line de Política e Gestão Educacional, Araraquara, v. 22, n. 3, p. 1065-1080, set./dez., 2018. Disponível em: https://periodicos.fclar.unesp.br/rpge/article/view/11874/7853. Acesso em: 25 set. 2020.

VARELA, Renata Cristina Bertolozzi; OLIVER, Fátima Corrêa. A utilização de Tecnologia Assistiva na vida cotidiana de crianças com deficiência. 2012. Disponível em:

https://www.scielosp.org/article/csc/2013.v18n6/1773-1784/pt/. Acesso em: 25 set. 2020. 
WAGNER, et al. Acessibilidade de pessoas com deficiência: um olhar da comunidade da periferia de Porto Alegre. Ciência em Movimento, ano 7, n. 23, 2010/1. Disponível em: https://www.metodista.br/revistas/revistas-ipa/index.php/RS/article/viewFile/94/58. Acesso em: 17 set. 2020.

Recebido em: 01/10/2021

Aprovado em: 28/10/2021

Publicado em: 05/11/2021 